\title{
WCVALEACH: WEIGHT AND COVERAGE BASED ENERGY EFFICIENT ADVANCED LEACH ALGORITHM
}

\author{
Prof Ankit Thakkar ${ }^{1}$ and Dr K Kotecha ${ }^{2}$ \\ ${ }^{1}$ Assistant Professor, Department of Computer Engineering, Institute of Technology, \\ Nirma University, Ahmedabad -382 481, Gujarat, India \\ ankit.thakkarenirmauni.ac.in \\ ${ }^{2}$ Director, Institute of Technology, Nirma University, Ahmedabad -382 481, Gujarat, \\ India \\ director.itenirmauni.ac.in
}

\begin{abstract}
Designing a protocol stack for wireless sensor network (WSN) is a challenging task due to energy, computational, communication and storage constraints. Energy spent for communication between sensor nodes dominates the energy spent for the computation [1]. Multi-hop short range communication between wireless sensor nodes is energy efficient compared to single-hop long range communication. Hierarchical clustering is one of the possible solutions to save energy of wireless sensor nodes. LEACH [16], Advanced LEACH (ALEACH) [14] and Weight based energy efficient Advanced LEACH (WALEACH) [20] are energy efficient hierarchical clustering routing protocols. In this paper we presented Weight and Coverage based Advanced LEACH routing protocol - WCVALEACH. WCVALEACH selects Cluster Head by assigning importance (weight) to different parameters used to select Cluster Head. It also creates the non-overlapped cluster regions to cover more number of member nodes. Both of these approaches makes WCVALEACH routing protocol energy efficient and improves life time of a wireless sensor network. Simulation results shown here verify that WCVALEACH not only improves network life time compared to ALEACH, LEACH and WALEACH algorithms but Packet Reception Rate (PRR) too.
\end{abstract}

\section{KEYWORDS}

Weight and Coverage based Advanced LEACH, Energy efficient, Wireless Sensor Network, Enhanced Packet Reception Rate (PRR), Clustering

\section{INTRODUCTION}

Wireless Sensor Network (WSN) getting popularity due to reduction in cost and size and advancement in Micro-Electro Mechanical System (MEMS). WSN getting attention due to their potential application in habitat monitoring system, forest fire detection, surveillance system, automated devices, structural health monitoring, Body Area Network (BAN) and other domains [2],[3]. Researchers getting interest in WSN because of many challenges and constraints associated with sensor networks which includes limited amount of energy, memory, communication and computational capabilities of a sensor node. To implement given task for a longer time, energy expenditure has to be done carefully by the sensor nodes. Energy required for communication is very high compared to computation and hence it should be carefully used to improve lifetime of a sensor network. Hierarchical clustering with data aggregation is one of the 
Computer Science \& Engineering: An International Journal (CSEIJ), Vol.2, No.6, December 2012

solutions to enhance the life time of a sensor network [4][5][6][7]. In most applications of a sensor network, sensor nodes are dropped from the helicopter in a hostile environment and sensor nodes will automatically establish connection with the neighboring nodes. In WSN each sensor node not only generates sensed data and to process collected data but also acts as a router to send data to Base Station (BS) where end user can get useful information of his interest. In cluster based algorithm, nodes are grouped and form a cluster. One of the nodes within a cluster acts as a Cluster Head $(\mathrm{CH})$ and other nodes within a cluster are called member nodes. Member nodes send their data to $\mathrm{CH}$ and $\mathrm{CH}$ forwards data to Sink node or Base Station (BS) directly or through other CHs using multi-hop communication.

Our algorithm is cluster based algorithm with the following objectives: To maximize the lifetime of a sensor network, to balance the energy depletion across the entire network and to enhance the Packet Reception Rate (PRR). Lifetime of a sensor network can be measured by three matrices: First Node Dies (FND), Half of the nodes Alive (HNA) and Last Node Dies (LND). In our protocol - WCVALEACH, we have introduced a new technique by assigning importance to different parameters to select the cluster head along with the creation of non-overlapping cluster region to make our algorithm energy efficient.

This paper is organized as follows: LEACH, ALEACH and WALEACH algorithms are discussed in Section 2, WCVALEACH algorithm is discussed in Section 3, Simulation results are presented in Section 4 and Concluding remarks are given in Section 5.

\section{RELATED WORK}

Energy being the most important constraint for WSN, now a days "Power Aware" routing techniques have been developed by many researcher [1], [8], [9], [10], [11], [12], [13]. In these techniques optimal routes are to be chosen which are energy-efficient and the node which is having highest energy will act as a cluster head. Our routing protocol, WALEACH, is also based on such technique by assigning weights to the different parameters used to select cluster head.

LEACH algorithm increases lifetime of the WSN by randomly rotating role of a $\mathrm{CH}$ among all the nodes. In LEACH, a node selects a random number between 0 and 1 . If the selected random number is less than the threshold value $T(n)$, then node declares itself as a $\mathrm{CH}$ for the current round. Threshold value $T(n)$ is given by equation 1 . LEACH ensures that each node becomes a cluster head only once in $1 / \mathrm{p}$ rounds, where $p$ is a desired percentage of $\mathrm{CH}$ during each round. LEACH does not consider the current state (energy level) of a node while electing it as a $\mathrm{CH}$.

$$
T(n)= \begin{cases}\frac{p}{1-p *\left(r \bmod \frac{1}{p}\right)} & \text { if } \mathrm{n} \in \mathrm{G} \\ 0 & \text { otherwise }\end{cases}
$$

ALEACH [14] is distributed energy efficient routing protocol which considers energy level of a node while electing CHs. ALEACH doesn't require to know the locations of the nodes. Also, there is no need to do global communication to elect $\mathrm{CH}$ which is required in $\mathrm{LEACH}-\mathrm{C}$ [15].

Like LEACH, ALEACH also works in rounds. Each round begins with Cluster Setup phase. During cluster setup phase a node will select a random number between 0 and 1 . If selected random number is less than threshold value $T(n)$ then node will declare itself as a cluster head where $T(n)$ is given by equation 2 .

$$
T(n)=G_{p}+C S_{p}
$$


where $G p$ and $C S p$ is given by equations 3 and 4 respectively. $G p$ and $C S p$ refer to general probability and current state probability.

$$
G_{p}=\frac{k}{N-k *\left(\mathrm{r} \bmod \frac{N}{k}\right)}
$$

where $\mathrm{k} / \mathrm{N}$ refers to the desired percentage of cluster heads during each round and Ecurrent and Emax is remaining energy and maximum energy of a node respectively.

$$
C S_{p}=\frac{E_{\text {current }}}{E_{\max }} X \frac{k}{N}
$$

ALEACH has considered energy level of a node to decide a node will become a $\mathrm{CH}$ during a particular round or not. Major drawback of ALEACH is proper importance (weights) is not given to general probability and current state probability. Main aim of our algorithm -WALEACH is to increase the lifetime of a WSN and increase the PRR by assigning proper weights to the general probability and current state probability.

WALEACH divides rounds into Cluster Set up phase and Steady Setup phase. Cluster Setup phase is used to elect cluster heads. During cluster setup phase each node selects a random number between 0 and 1 . If selected number is less than threshold value $T(n)$, a node elects itself as a cluster head for the current round, where $T(n)$ is given by equation 2 . The general probability and current state probability is given by equation 5 and equation 6 respectively. In these equations $w$ is used to assign weight (importance) to general probability and current state probability.

$$
\begin{gathered}
G_{p}=\frac{N-(w * k)}{N} X \frac{k}{N-k *\left(\mathrm{r} \bmod \frac{N}{k}\right)} \\
C S_{p}=\frac{w * k}{N} X \frac{E_{\text {current }}}{E_{\max }}
\end{gathered}
$$

CVLEACH[21] is a Coverage based LEACH algorithm. It creates non-overlapping cluster regions to cover more member nodes by the cluster heads to conserve energy of nodes and enhances life time of a sensor network.

\section{WCVALEACH: WEIGHT AND COVERAGE BASED ADVANCED LEACH ROUTING PROTOCOL}

ALEACH has considered energy level of a node to decide a node will become a $\mathrm{CH}$ during a particular round or not. Major drawback of ALEACH is proper importance (weight) is not given to general probability and current state probability. WALEACH assigns importance to general probability and current state probability. Main aim of our algorithm - WCVALEACH is to increase the lifetime of a WSN and to increase PRR by assigning proper weights to the general probability and current state probability. It also creates non-overlapping cluster regions as does in CVLEACH [21] to further increase the lifetime of WSN. 
WCVALEACH divides rounds into Cluster Set up phase and Steady Setup phase. Cluster Setup phase is used to elect cluster heads. During cluster setup phase each node selects a random number between 0 and 1 . If selected number is less than threshold value $T(n)$, a node elects itself as a cluster head for the current round, where $T(n)$ is given by equation 2 . The general probability and current state probability is given by equation 5 and equation 6 respectively. In these equations $w$ is used to assign weight (importance) to general probability and current state probability.

When any node receives Cluster Head announcement, it withdraws its participation to be elected as a cluster head for the current round. This helps to create non-overlapping cluster regions, and covers more number of member nodes. Cluster Set up phase of WCVALEACH algorithm is shown in Algorithm 1 and Steady Set up phase is shown in Algorithm 2 which is used by member nodes to send data to their cluster heads.

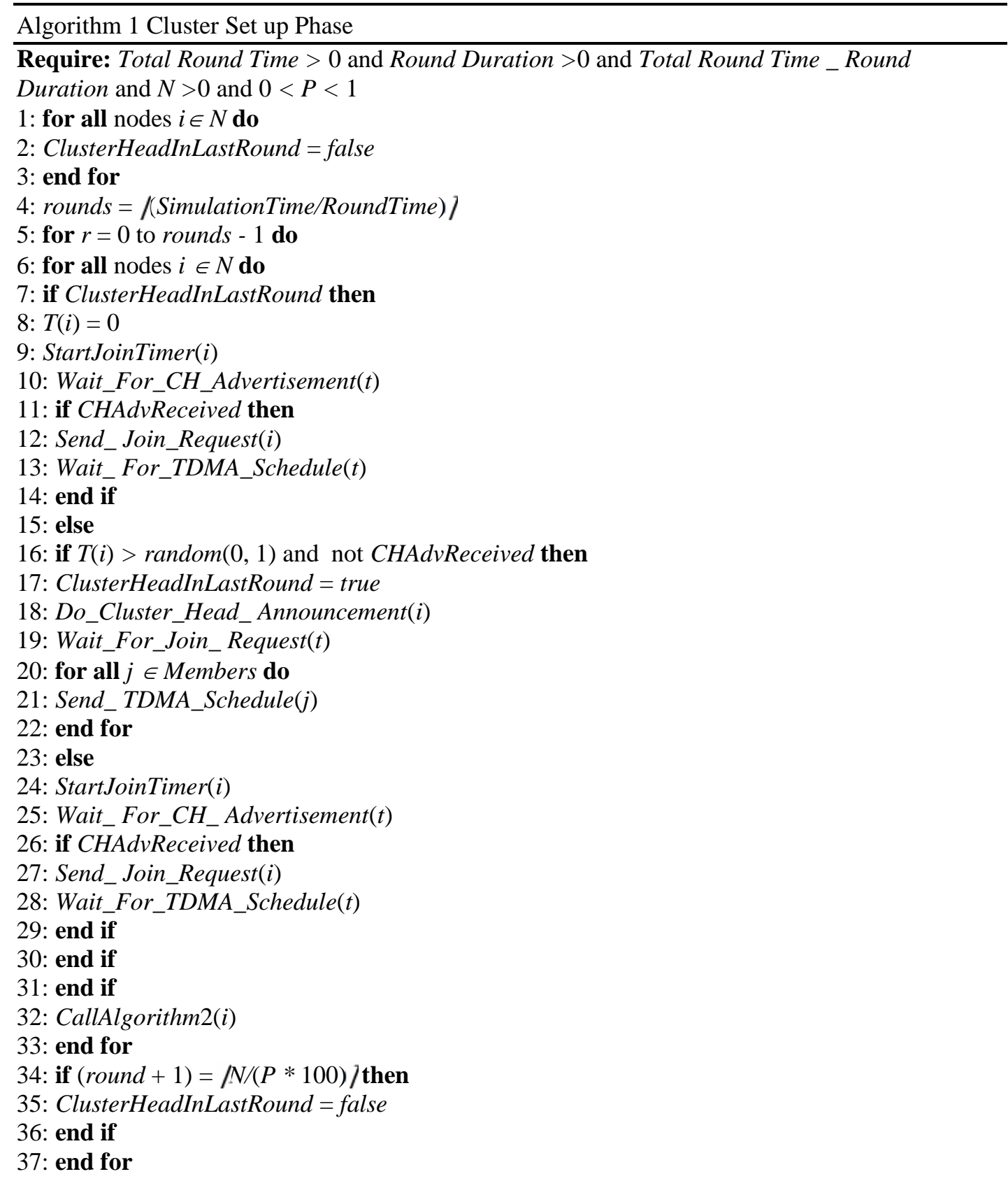


Algorithm 2 Steady-State Phase

Require: $i$

1: if $t<$ RoundTime then

2: if $i \in C H$ then

3: for all $j \in$ Members $(i)$ do

4: Collect_Data_From_Member_Nodes $(j)$

5: end for

6: Send_Aggregate_Data_To_Sink_By_CH(i)

7: else

8: if $t<$ Scheduled Start Time or $t>$ Scheduled End Time then

9: Sleep_Node( $i)$

10: else

11: Send_Data_To_CH_By_Member(i)

12: end if

13: end if

14: end if

\section{RESULTS AND DISCUSSION}

The network model used in WCVALEACH is having following properties as mentioned in [14],[15],[16],[17],[20].

- BS consists of high level of energy and located far away from sensor nodes.

- All nodes within the network are immobile.

- All nodes are uniform.

- Each node is able to control its transmission power as and when required.

- Each node sense environment at a fixed rate and have some data to send to BS or $\mathrm{CH}$.

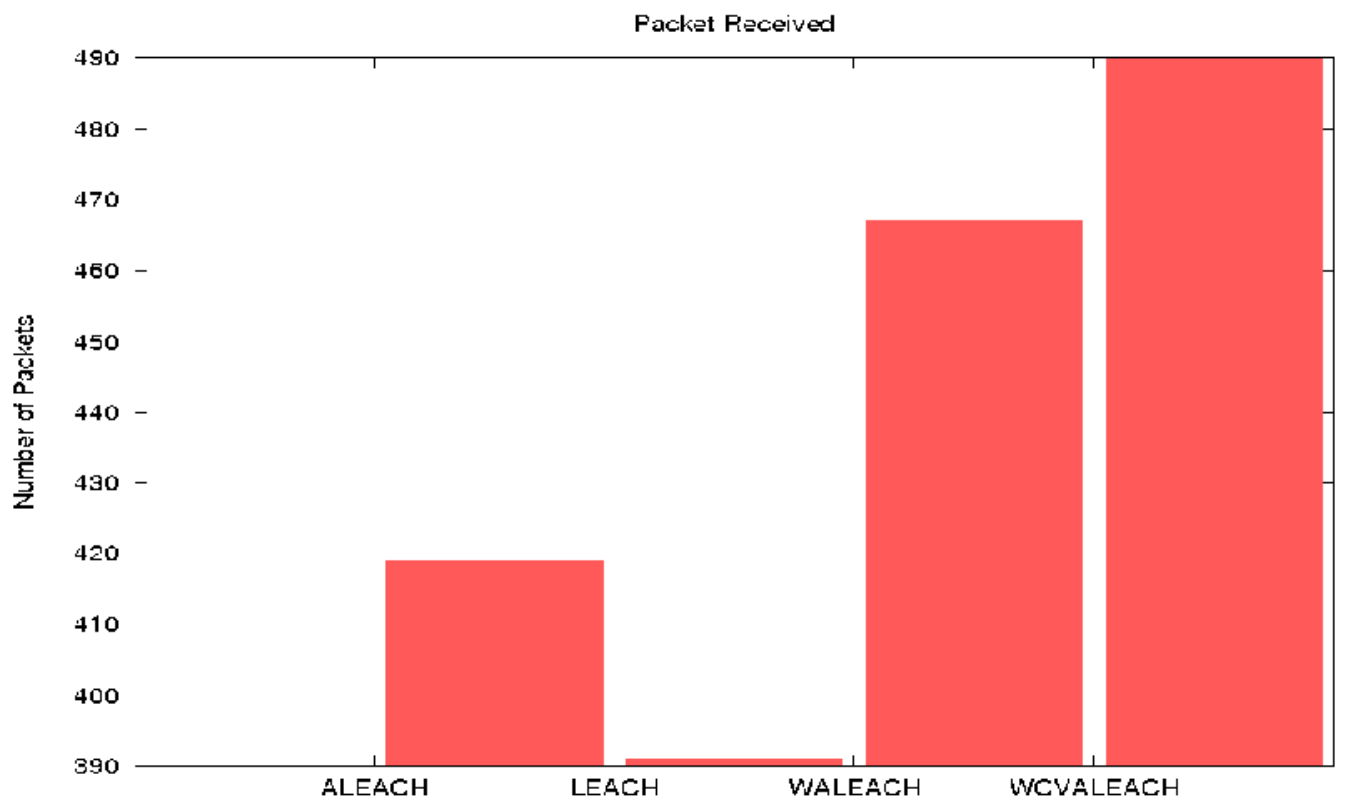

Figure 1. Number of Packets Received 


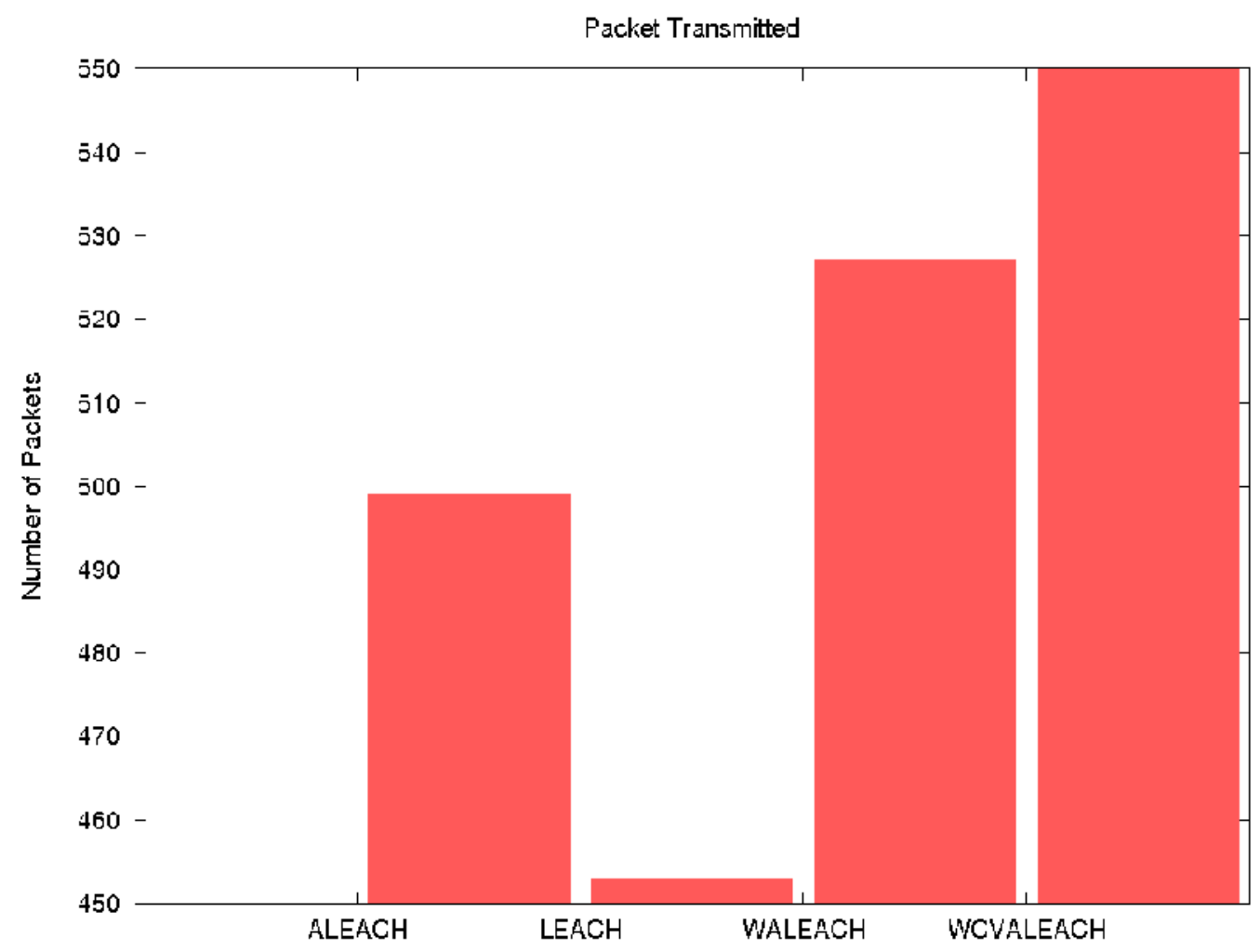

Figure 2. Number of Packets Transmitted

We have investigated performance of WCVALEACH against LEACH, ALEACH and WALEACH algorithm using Castalia simulator [18]. Castalia can be used for simulation of Wireless Sensor Networks (WSN), Body Area Networks (BAN) and networks of low-power embedded devices. It is used to test distributed algorithms and/or protocols in realistic wireless channel and radio models, with a realistic node behavior especially relating to access of the radio [18]. A network of size $500 \mathrm{mx} 500 \mathrm{~m}$ is created with randomly distributed 100 sensor nodes to test performance of WALEACH against LEACH and ALEACH. Each node has CC2420 as transceiver. Each node constantly draws $6 \mathrm{~mW}$ and sensing device within the node draws $0.02 \mathrm{~mW}$. The transceiver energy parameters are set as: ETx $=46.2 \mathrm{~mW}$ to do cluster head announcement, to send cluster join message and to send data by member nodes to their respective cluster heads; $\mathrm{ETx}=57.42 \mathrm{~mW}$ to send data by the non cluster member nodes and cluster heads to Base Station. A transceiver of node can be in one of the three states: RX (receive), TX (transmit) or SLEEP, if node is alive. Transceiver takes the $194 \mu \mathrm{sec}$ to change from SLEEP state to either

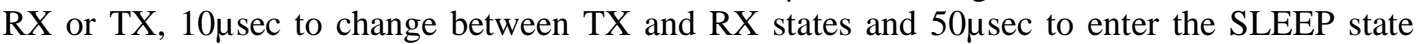
[18]. Transition to SLEEP state from RX or TX consumes $1.4 \mathrm{~mW}$ while any other transition consumes $62 \mathrm{~mW}[18]$. 
Table 1. Simulation Parameters

\begin{tabular}{|c|c|}
\hline Parameter & Value \\
\hline Node Deployment Area & $500 \mathrm{~m} \times 500 \mathrm{~m}$ \\
\hline Number of Nodes & 100 \\
\hline Initial Energy & 18720 Joules \\
\hline Energy to do $\mathrm{CH}$ announcement & \multirow{3}{*}{$46.2 \mathrm{~mW}$} \\
\hline Energy to transmit data to $\mathrm{CH}$ & \\
\hline Energy to send CH Join Message & \\
\hline Energy to transmit BS & $57.42 \mathrm{~mW}$ \\
\hline Rate of Sensory Data generation & Every 2 Seconds \\
\hline Round Duration & 20 Seconds \\
\hline Simulation Time & 1000 Seconds \\
\hline Operating power of a Node & $6 \mathrm{~mW}$ \\
\hline Operating power of a sensing device & $0.02 \mathrm{~mW}$ \\
\hline Size of Control and Data messages & 30 Bytes \\
\hline Expected percentage of $\mathrm{CH}$ per round & $5 \%$ \\
\hline Weight Factor (w) & 3.81 \\
\hline Initial Energy (for FND,HND,LND) & 6 Joules \\
\hline
\end{tabular}

Size of control and data message is set to 30 bytes. Every 2 -seconds sensor will report sensed data to the node itself. Round time is fixed to 20 -seconds. Each node has given 18720 Joules of initial energy which is equivalent to energy of two AA batteries [18]. $p$ is set to 5\% in Equation 1. $w$ is set to 3.81 in Equations 5 and 6. This value of $w$ gives optimal performance of WALEACH and WCVALEACH. This value of $w$ is verified through experiments. Simulation parameters is listed in Table 1.

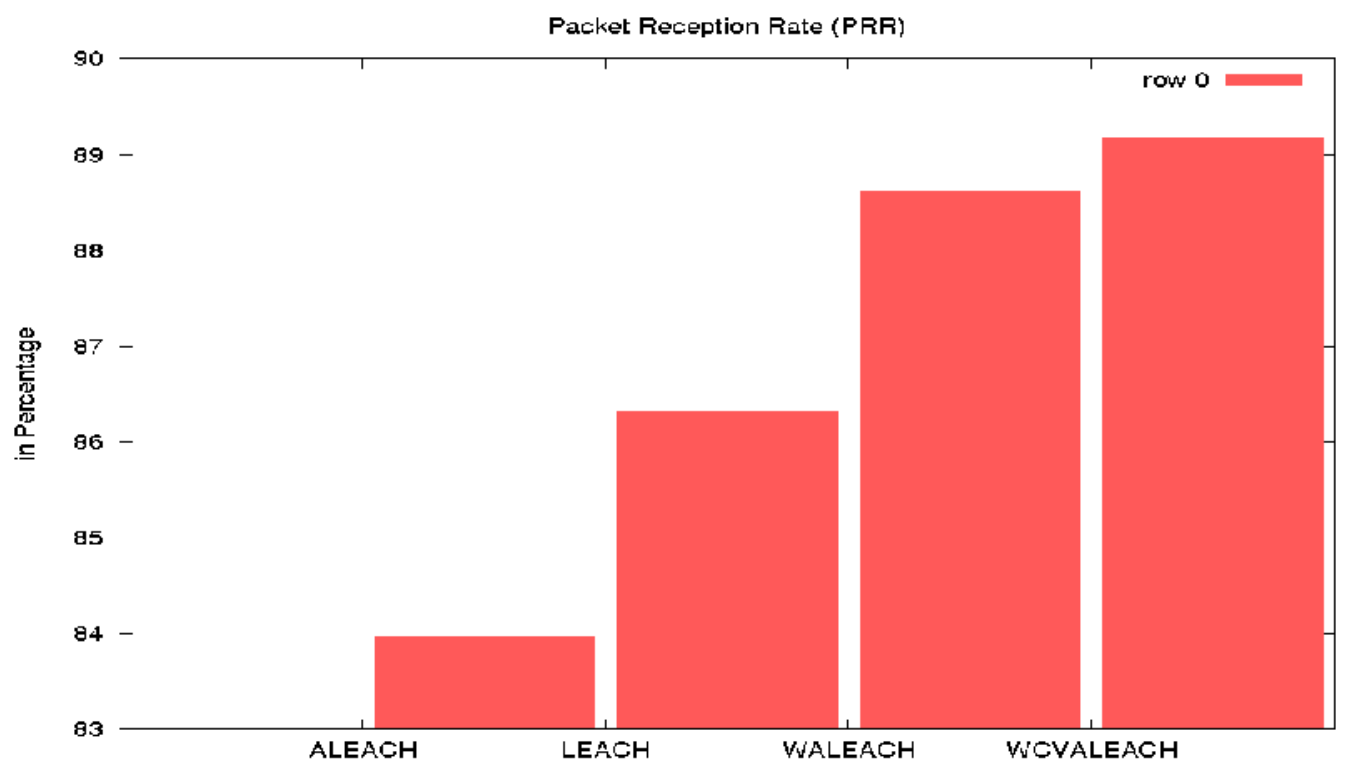

Figure 3. Packet Reception Rate (in Percentage) 
Computer Science \& Engineering: An International Journal (CSEIJ), Vol.2, No.6, December 2012

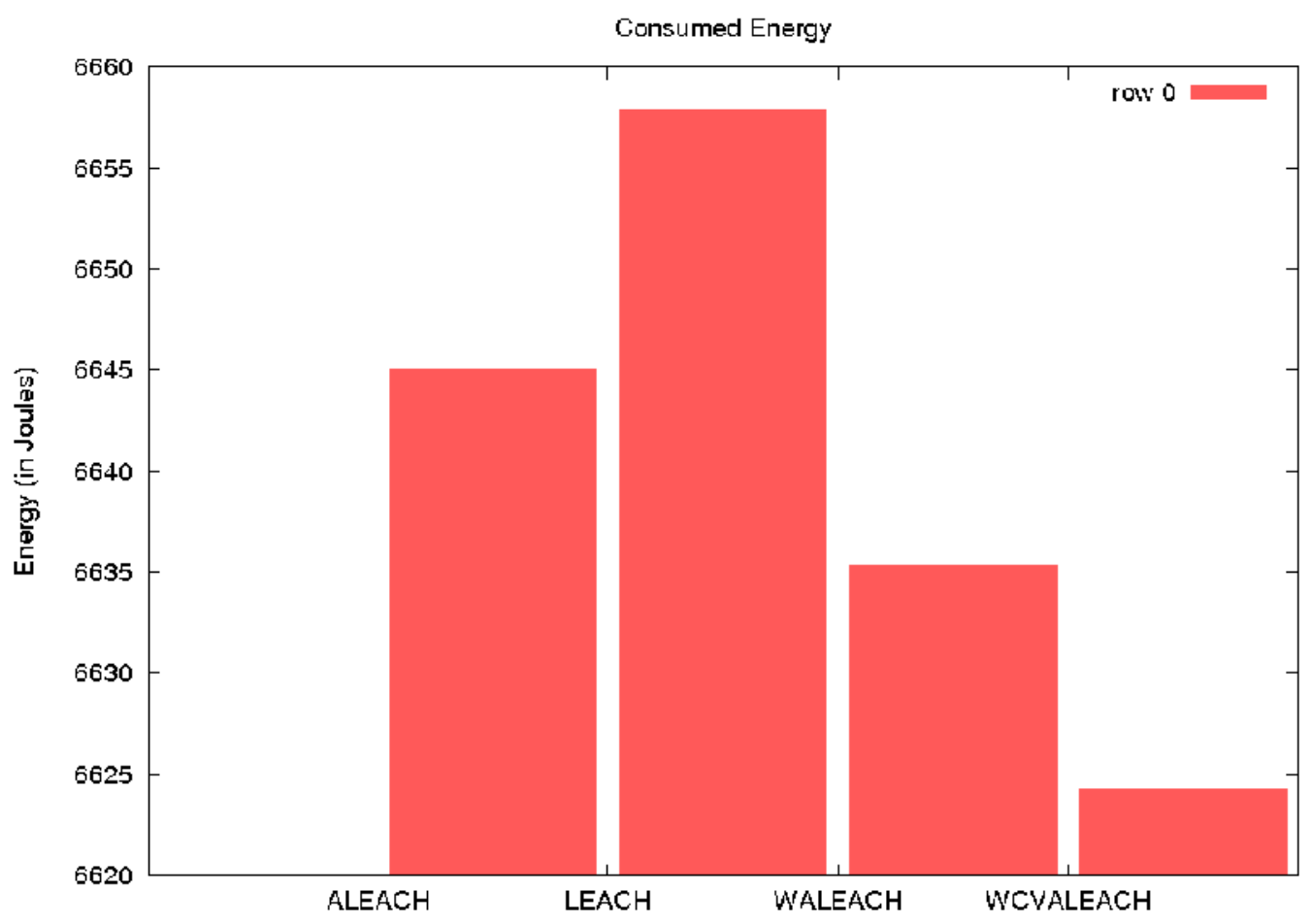

Figure 4. Total Energy Consumption (in Joules)

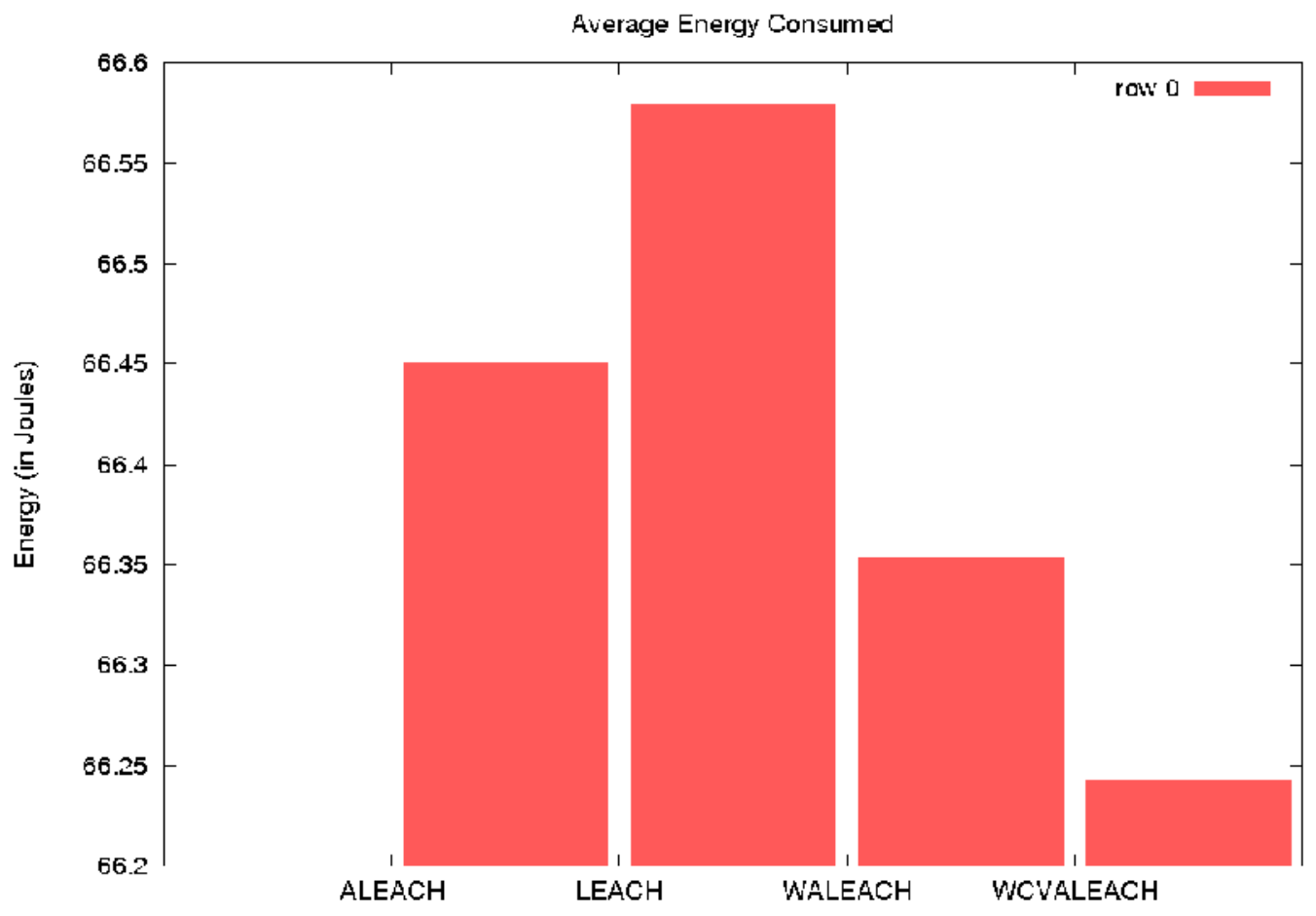

Figure 5. Average Energy Consumption per Node (in Joules) 
Computer Science \& Engineering: An International Journal (CSEIJ), Vol.2, No.6, December 2012

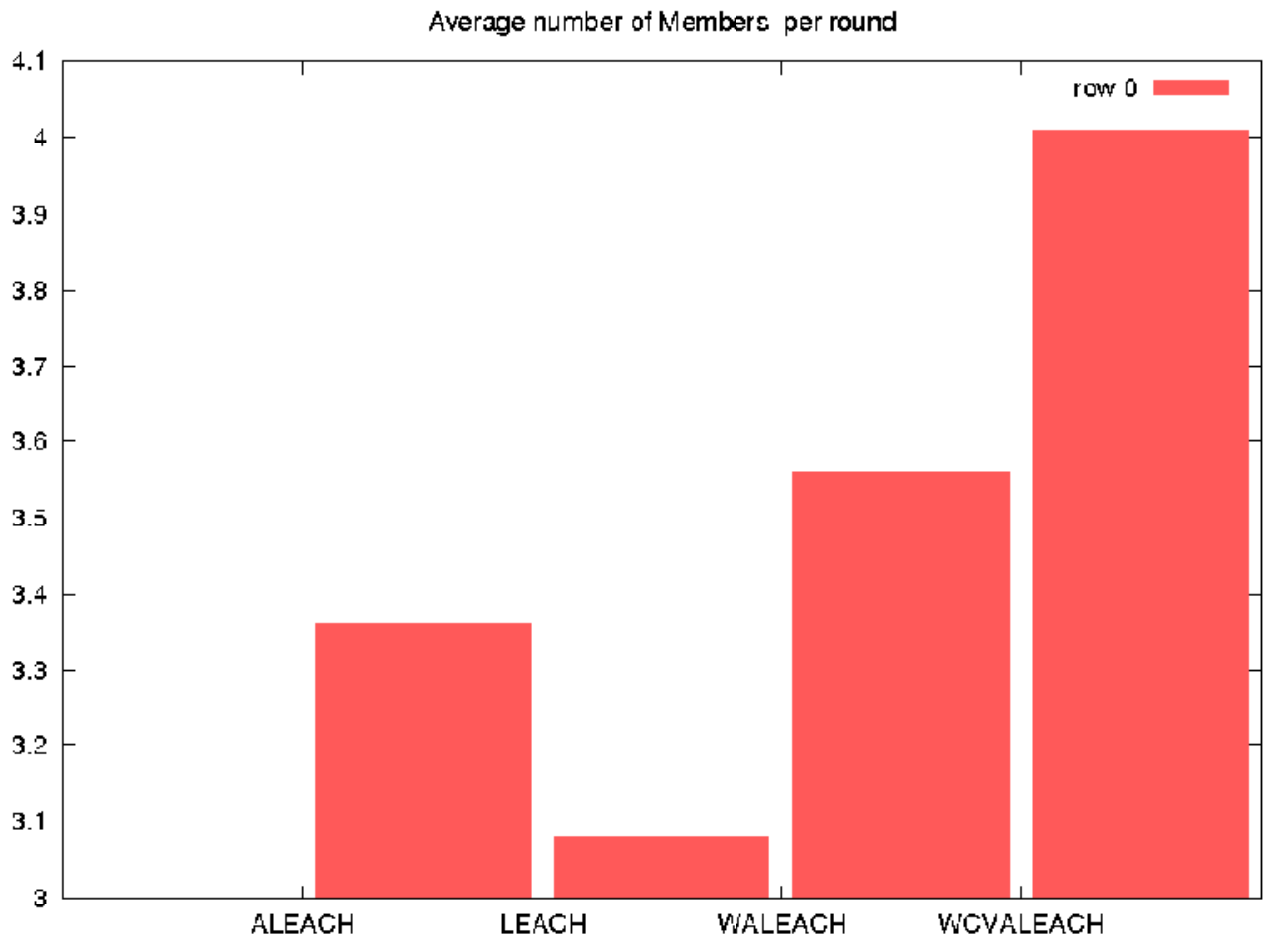

Figure 6. Average Number of Member Nodes per Round

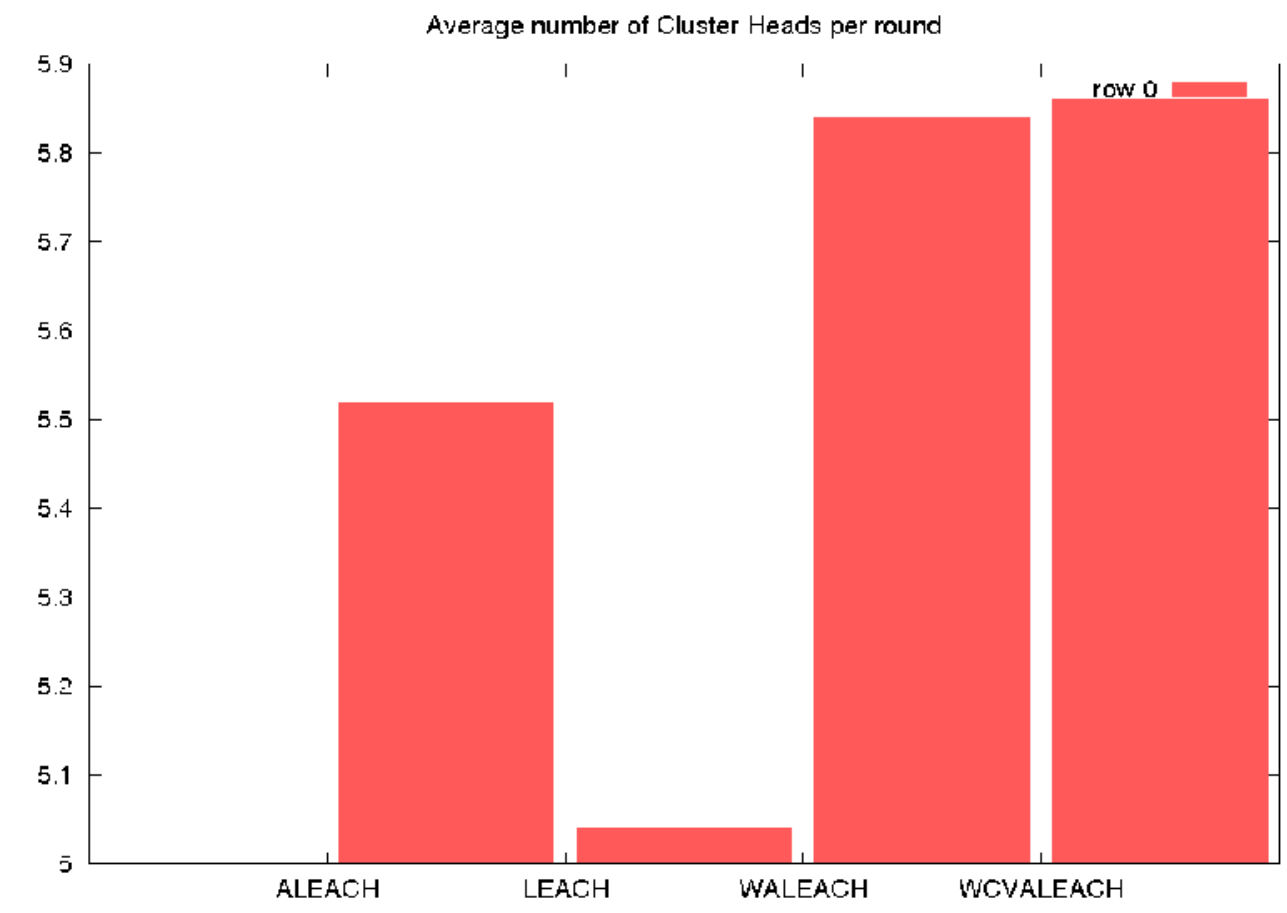

Figure 7. Average Number of Cluster Heads per Round 
Computer Science \& Engineering: An International Journal (CSEIJ), Vol.2, No.6, December 2012

We have run simulation for 1000 seconds to investigate following performance metrics: Packet Reception Rate: It represents the ratio of number of packet received over number of packet transmitted; Energy consumption: It represents total energy consumption of all nodes, and Average energy consumption per node: It represents the average energy expenditure of a node. Coverage: Average number of members covered by all cluster heads per round; Fitness of Cluster Head: It represents average number of cluster heads; Network Life Time: Network life time can be measured in terms of First Node Dies (FND), Half of the Nodes Alive (HNA) and Last Node Dies (LND) and total life time of all nodes. Total number of packets received and transmitted is shown in figure 1 and 2 respectively. Total packets received for LEACH, ALEACH, WALEACH and WCVLEACH are 391, 419, 467 and 490 while total packets transmitted for LEACH, ALEACH, WALEACH and WCVALEACH are 453, 499, 527 and 550 respectively. Packet reception rate for LEACH, ALEACH, WALEACH and WCVALEACH is found $86.314 \%$, $83.968 \%, 88.615 \%$ and $89.09 \%$ respectively and is shown in Figure 3. Total energy consumption of all nodes and average energy consumption per node for LEACH, ALEACH, WALEACH and WCVALEACH is shown in figures 4 and 5 respectively. Total energy consumption of all nodes is 6657.897 Joules, 6645.132 Joules, 6636.108 Joules and 6624.264 Joules and average energy consumption per node is 66.579 Joules, 66.451 Joules, 66.361 Joules and 66.243 Joules recorded for LEACH, ALEACH, WALEACH and WCVALEACH algorithm respectively. For the simulation, $p$ is set to $5 \%$ in equation 1 and $w$ is set to 3.81 in equations 5 and 6 . Number of nodes which are covered by the cluster heads and average number of cluster heads is shown in Figure 6 and Figure 7 respectively. An average member of all clusters during each round is shown in Figure 6 and it is 3.08, 3.36, 3.56 and 4.01 for LEACH, ALEACH, WALEACH and WCVALEACH algorithm. It can be seen from Figure 7 that average number of cluster heads per round for LEACH, ALEACH, WALEACH and WCVALEACH is 5.04, 5.52, 5.84 and 5.86. Energy required to communicate with cluster heads is very less compared to energy required to communicate with base station. Hence, energy conservation for WCVALEACH is better than ALEACH, LEACH and WALEACH algorithms.

There are three major matrices which can be used to measure lifetime of sensor nodes: First Node Dies - FND, Half of the Nodes Alive - HNA, and Last Node Dies - LND [19]. To test these matrices, 6 Joules of initial energy is given to each node and FND, HNA and LND recorded for LEACH, ALEACH, WALEACH and WCVALEACH which is shown in Table 2. It can be seen from the Table 2 that WCVALEACH is better than LEACH, ALEACH and WALEACH algorithms in terms of FND, HNA and LND. Total life time all the nodes of WCVALEACH is $4.867 \%$ better than LEACH, 3.954\% better than ALEACH and $1.848 \%$ better than WALEACH when only 6 Joules of initial energy is given to each node. Comparative of performance matrices simulated for ALEACH, WALEACH, LEACH and WCVALEACH is shown in Table 3.

Table 2. FND, HNA, LND and Network life time (initial Energy 6 Joules/Node)

\begin{tabular}{|l|l|l|l|l|}
\hline Algorithm & $\begin{array}{l}\text { FND } \\
\text { (in Seconds) }\end{array}$ & $\begin{array}{l}\text { HNA } \\
\text { (in Seconds) }\end{array}$ & $\begin{array}{l}\text { LND } \\
\text { (in Seconds) }\end{array}$ & $\begin{array}{l}\text { Network Life Time (in } \\
\text { Seconds) }\end{array}$ \\
\hline ALEACH & 88.248 & 89.076 & 103.677 & 9176.344 \\
\hline WALEACH & 88.268 & 88.999 & 116.169 & 9377.580 \\
\hline LEACH & 88.243 & 88.855 & 103.710 & 9089.122 \\
\hline WCVLEACH & $\mathbf{8 8 . 2 7 3}$ & $\mathbf{9 0 . 5 0 9}$ & $\mathbf{1 2 7 . 2 4 2}$ & $\mathbf{9 5 5 4 . 1 5 0}$ \\
\hline
\end{tabular}


Computer Science \& Engineering: An International Journal (CSEIJ), Vol.2, No.6, December 2012

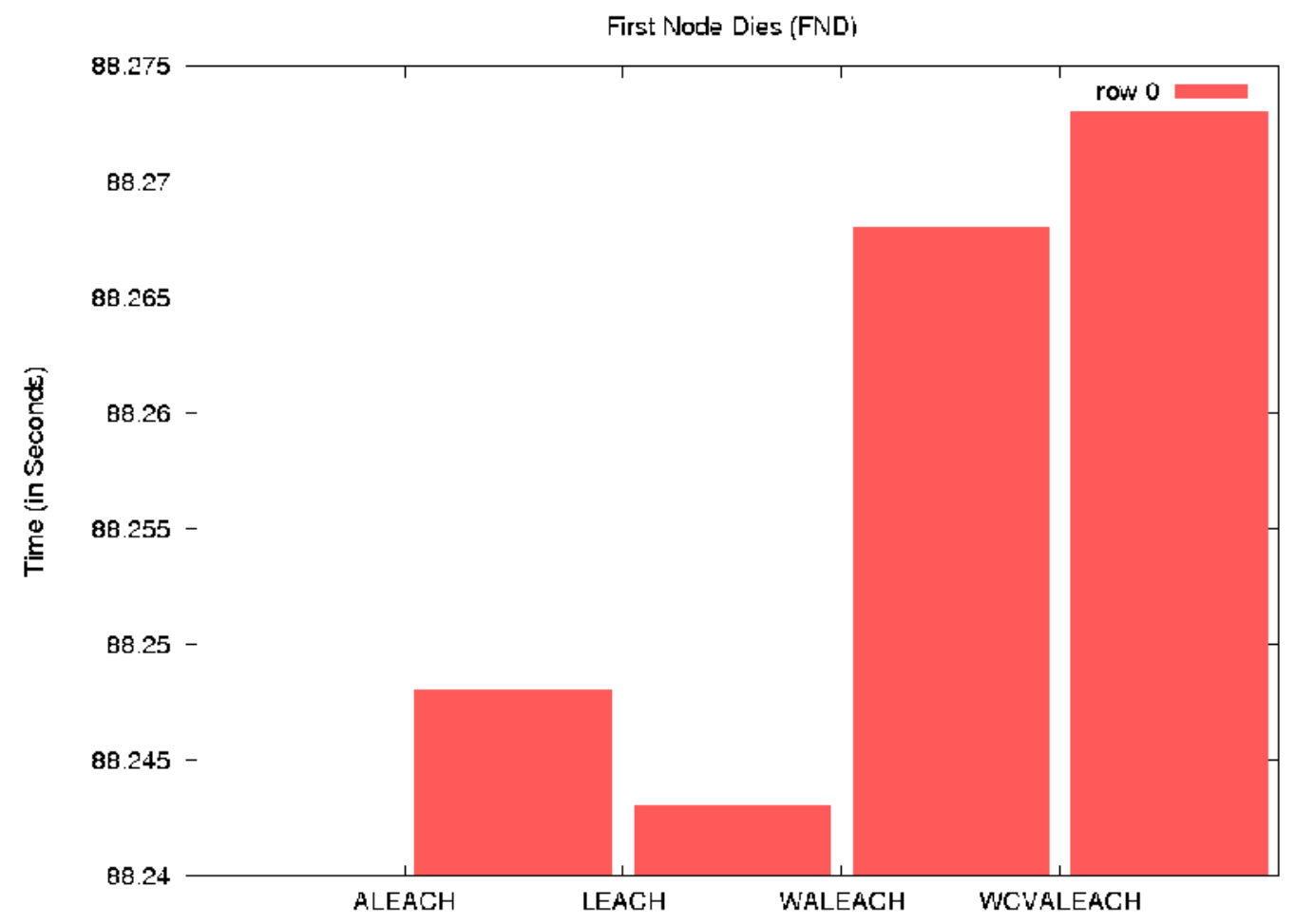

Figure 9. First Node Dies (FND)

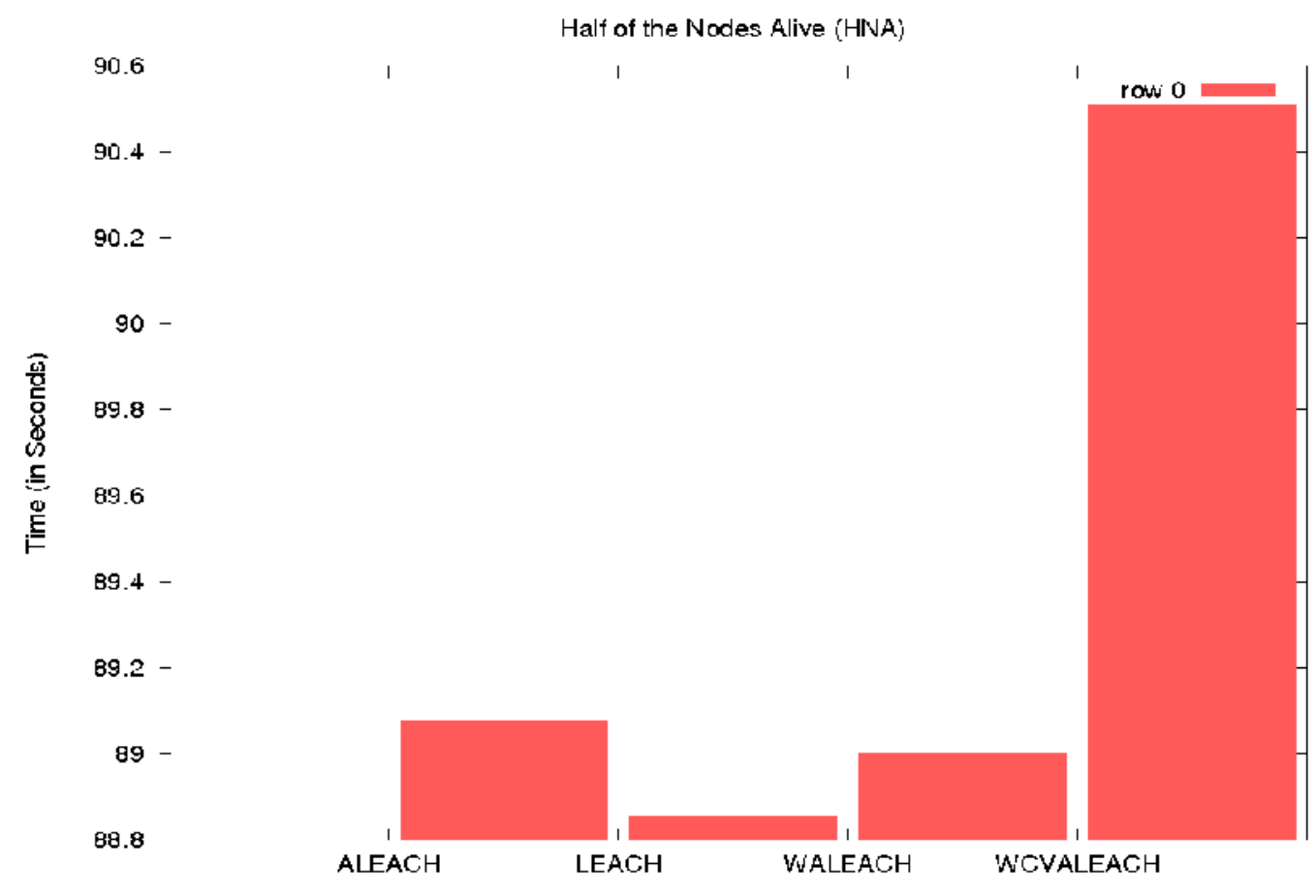

Figure 10. Half of the Nodes Alive (HNA) 
Computer Science \& Engineering: An International Journal (CSEIJ), Vol.2, No.6, December 2012

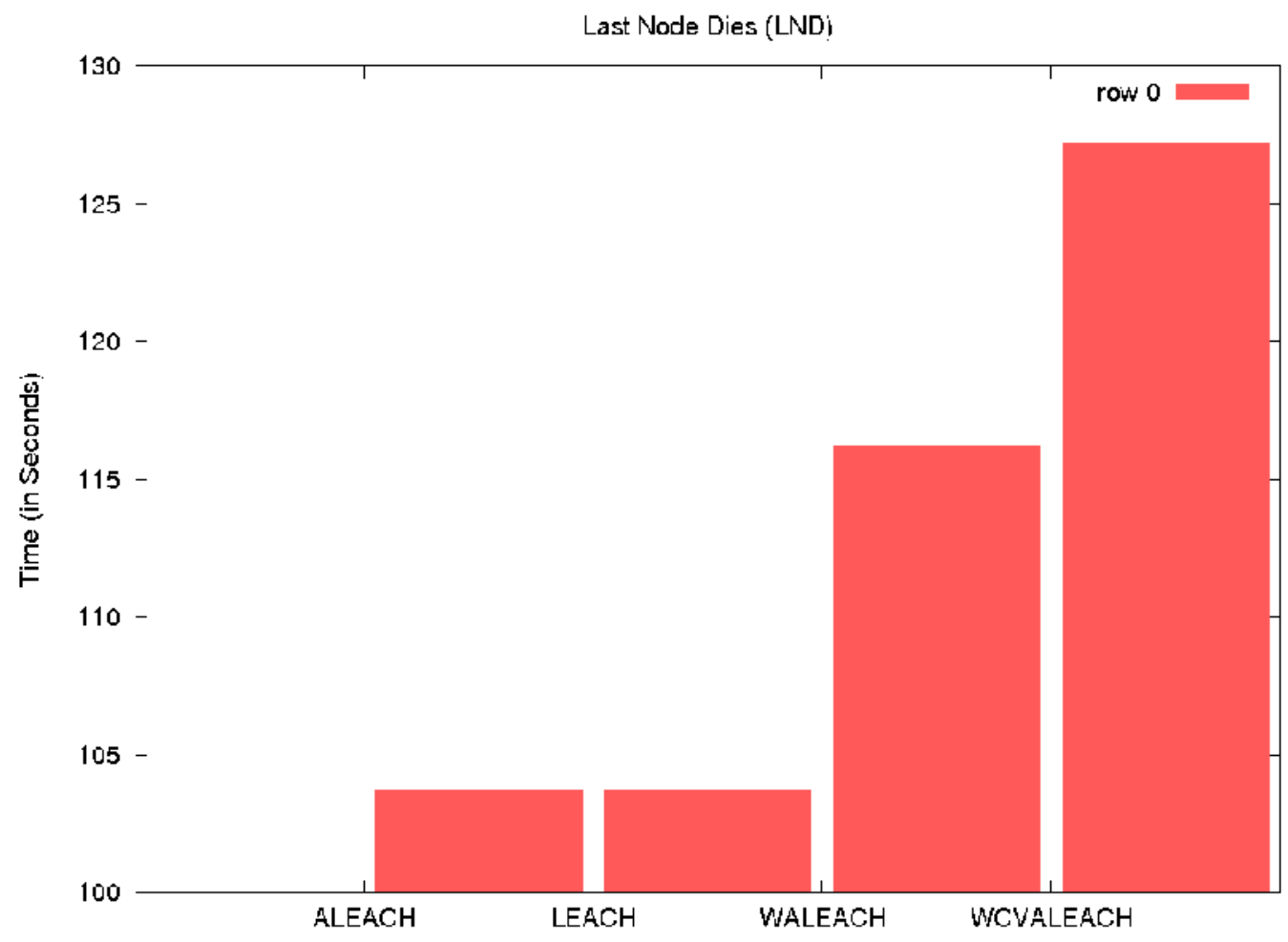

Figure 11. Last Node Dies (LND)

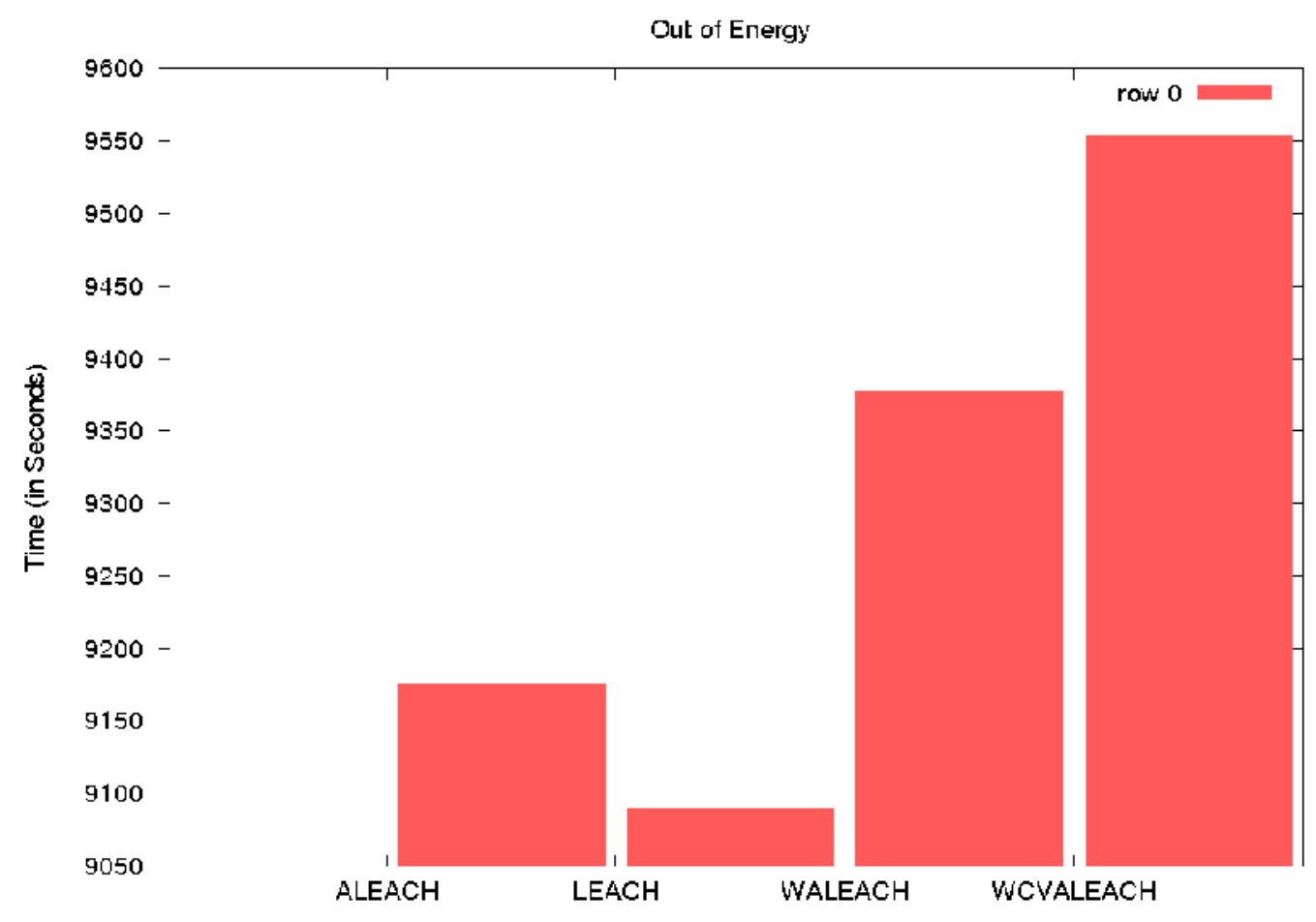

Figure 12. Total Life Time of the Network with Initial Energy 6 Joules/Node 
Computer Science \& Engineering: An International Journal (CSEIJ), Vol.2, No.6, December 2012

Table 3. Comparative of Performance Matrices

\begin{tabular}{|l|l|l|l|l|}
\hline $\begin{array}{l}\text { Performance } \\
\text { Matrix }\end{array}$ & LEACH & ALEACH & WALEACH & WCVALEACH \\
\hline $\begin{array}{l}\text { Number of Packets } \\
\text { Transmitted }\end{array}$ & 453 & 499 & 527 & $\mathbf{5 5 0}$ \\
\hline $\begin{array}{l}\text { Number of Packets } \\
\text { Received }\end{array}$ & 391 & 419 & 467 & $\mathbf{4 9 0}$ \\
\hline $\begin{array}{l}\text { Packet Reception } \\
\text { Rate }\end{array}$ & $86.314 \%$ & $83.968 \%$ & $88.615 \%$ & $\mathbf{8 9 . 0 9 \%}$ \\
\hline $\begin{array}{l}\text { Total Energy } \\
\text { Consumption }\end{array}$ & $\begin{array}{l}6657.897 \\
\text { Joules }\end{array}$ & $\begin{array}{l}6645.132 \\
\text { Joules }\end{array}$ & $\begin{array}{l}6636.108 \\
\text { Joules }\end{array}$ & $\mathbf{6 6 2 4 . 2 6 4 ~ J o u l e s ~}$ \\
\hline $\begin{array}{l}\text { Average Energy } \\
\text { Consumption per } \\
\text { Node }\end{array}$ & $\begin{array}{l}66.579 \\
\text { Joules }\end{array}$ & $\begin{array}{l}66.451 \\
\text { Joules }\end{array}$ & 66.361 Joules & $\mathbf{6 6 . 2 4 3}$ Joules \\
\hline $\begin{array}{l}\text { Average Number of } \\
\text { Cluster Heads per } \\
\text { Round }\end{array}$ & $\mathbf{5 . 0 4}$ & 5.52 & 5.84 & 5.86 \\
\hline $\begin{array}{l}\text { Average Number of } \\
\text { Members per Round }\end{array}$ & 3.08 & 3.36 & 3.56 & $\mathbf{4 . 0 1}$ \\
\hline
\end{tabular}

\section{CONCLUSION}

WCVALEACH is distributed and energy efficient algorithm. Neither it requires location information of a node nor does it require exchange of communication messages to decide the Cluster Head. It increases the lifetime of a sensor network which we have verified using FND, HNA and LND matrices. It also increases Packet Reception Rate (PRR). WCVALEACH does energy conservation by assigning weight as WALEACH and by creating non-overlapping cluster regions as CVLEACH.

\section{REFERENCES}

[1] S. Rizvi, K. Patel, and C. Patel, "Use of self-adaptive methodology in wireless sensor networks for reducing the energy consumption," in Proceedings of Novel Algorithm and Telecommunications, Automations and Industrial Electronics, 2008, pp. 519-525.

[2] A. Natarajan, M. Motani, B. de Silva, K. Yap, and K. C. Chua,’'Investigating network architectures for body sensor networks," in Network Architectures, G. Whitcomb and P. Neece, Eds. Dayton, OH:Keleuven Press, 2007, pp. 322-328.

[3] D. Culler, D. Estrin, and M. Srivastava, "Overview of sensor networks," IEEE Comput., vol. 37, no. 8 (Special Issue on Sensor Networks), pp. 41-49, 2004.

[4] I. F. Akyildiz, W. Su, Y. Sankarasubramaniam, and E. Cayirci,"Wireless sensor networks: A survey," Comm. ACM, vol. 38, no. 4, pp. 393-422, 2002.

[5] I. F. Akyildiz, T. Melodia, and K. R. Chowdhury, "A survey on wireless multimedia sensor networks," Computer Networks, vol. 51, no. 4, pp. 921-960, 2007.

[6] J. N. Al-Karaki and A. E. Kamal, "Routing techniques in wireless sensor networks: A survey," IEEE Wireless Communications, December 2004.

[7] K. Akkaya and M. Younis, "A survey on routing protocols for wireless sensor networks," Ad Hoc Networks, vol. 3, pp. 325-349, 2005.

[8] K. T. Kim and H. Y. Youn, "Energy-driven adaptive clustering hierarchy (edach) for wireless sensor networks," 2005.

[9] S. Park and M. Srivastava, "Power aware routing in sensor networks using dynamic source routing," in ACM MONET Special Issue on Energy Conserving Protocols in Wireless Networks, 1999. 
Computer Science \& Engineering: An International Journal (CSEIJ), Vol.2, No.6, December 2012

[10] S. Singh, M. Woo, , and C. Raghavendra, "Power-aware routing in mobile ad hoc networks," in Proceedings 4th Annual ACM/IEEE International Conference Mobile Computing and Networking (MobiCom), October 1998, pp. 181-190.

[11] M. Liu, J. Cao, G. Chen, and X. Wang, "An energy-aware routing protocol in wireless sensor networks," Sensors, vol. 9, 2009.

[12] D. Kandris, P. Tsioumas, A. Tzes, G. Nikolakopoulos, and D. D. Vergados, "Power conservation through energy efficient routing in wireless sensor networks," Sensors, vol. 9, pp. 7320-7342, September 2009.

[13] K. Iwanicki and M. van Steen, "On hierarchical routing in wireless sensor networks," in International Conference on Information Processing in Sensor Networks -IPSN '09. San Francisco, California, USA: IEEE Computer Society, APRIL 2009, pp. 133-144.

[14] M. S. Ali, T. Dey, and R. Biswas, "Aleach: Advanced leach routing protocol for wireless microsensor networks," in Proceedings of 5th International Conference on Electrical and Computer Engineering, ICECE. Dhaka, Bangladesh: IEEE, December 2008.

[15] W. B. Heinzelman, A. P. Chandrakasan, and H. Balakrishnan, "An application-specific protocol architecture for wireless microsensor networks," IEEE TRANSACTIONS ON WIRELESS COMMUNICATIONS, 2002.

[16] W. Heinzelman, A. Chandrakasan, and H. Balakrishnan, "Energy-efficient communication protocol for wireless sensor networks," in Proceedings of the Hawaii International Conference System Sciences, Hawaii, 2000.

[17] S. D. Muraganathan, D.C. MA, R. I. Bhasin and A. O. Fapojumo, "A centralized energy-efficient routing protocol for wireless sensor networks", IEEE Radio Communication, vol. 43, March 2005.

[18] "Castalia simulator," 2011, http://castalia.npc.nicta.com.au.

[19] Wireless Sensor Networks - A networking perspective. A JOHN WILEY and SONS, INC., PUBLICATION, 2009.

[20] A. Thakkar and K Kotecha, "WALEACH: Weight based energy efficient Advanced LEACH algorithm”, 4th International Workshop on Wireless and Mobile Networks (WiMo - 2012), vol. 2, No 4, October 2012.

[21] A. Thakkar and K Kotecha, "CVLEACH: Coverage based energy efficient LEACH algorithm", International Journal of Computer Science \& Network (IJCSN), vol. 1, No 3, June 2012.

\section{Authors}

Prof Ankit Thakkar - is working as Assistant Professor at Institute of Technology, Nirma University, Ahmedabad - 382 481, Gujarat, India. He is pursuing Ph. D. in the area of Wireless Sensor Networks. He has received "Gold Medal" for securing highest CPI among all graduating students of M.Tech. Computer Science \& Engineering 2007-09 batch from Institute of Technology, Nirma University, Ahmdedabad. His area of interest includes Wireless Sensor Networks, Embedded Systems, Image Processing, Real-time Systems and Data Structures and Algorithms.

Dr K Kotecha - is working as Director, Institute of Technology, Nirma University, Ahmedabad -382 481, Gujarat, India. He did his M.Tech. and Ph.D. from IIT, Bombay. His area of interest includes Analysis of Algorithms, Optimization Techniques and Sensor Networks 